\title{
Patrones temporales de participación en MOOC. Estudio de un MOOC de lenguas
}

\section{(Temporal patterns of MOOC participation. Case study of a language MOOC)}

\author{
Juan José del Peral Pérez \\ Universidad Nacional de Educación a Distancia, UNED (España)
}

DOI: http://dx.doi.org/10.5944/ried.22.2.23109

\section{Cómo referenciar este artículo:}

Del Peral, J. J. P. (2019). Patrones temporales de participación en MOOC. Estudio de un MOOC de lenguas. RIED. Revista Iberoamericana de Educación a Distancia, 22(2), pp. 287-303. doi: http://dx.doi.org/10.5944/ried.22.2.23109

\section{Resumen}

Aunque los Massive Open Online Courses (MOOC) se han convertido en una herramienta de aprendizaje continuo en la sociedad actual, las elevadas tasas de abandono son uno de sus principales problemas. El trazado de perfiles de usuario y la identificación de las características intrínsecas de cada perfil permite adaptar el diseño de los MOOC para adecuarlo a los patrones de uso de los estudiantes con el objetivo de incrementar las tasas de compleción de los cursos. Los perfiles de usuario que se han identificado hasta la fecha se han basado en el nivel de vinculación de los participantes del MOOC y la preferencia por un tipo u otro de actividades. Este estudio tiene como objetivo el trazado de perfiles de participantes en los MOOC en función del uso temporal del curso por parte de los mismos, tomando en cuenta tanto el número de sesiones como la repetición de franjas horarias por parte de los usuarios, con el objetivo de contrastar los rasgos fundamentales de cada tipo de estudiante. Para ello se ha utilizado la información contenida en los ficheros de log generados durante el desarrollo de la tercera edición del MOOC "Inglés Profesional / Professional English", implantado en la plataforma de UNED Abierta. A partir de los resultados obtenidos se puede concluir que la pertenencia a cualquiera de los distintos perfiles que completan el MOOC no implica un mayor desempeño en el curso ni nota mayor.

Palabras clave: MOOC; LMOOC; MOOC de lenguas; enseñanza de lenguas; enseñanza a distancia; participación de los estudiantes. 


\begin{abstract}
Although in today's society Massive Open Online Courses (MOOC) have become one of the main tools for continuous learning, one of their main problems is the high dropout rates. The mapping of user profiles and the identification of the intrinsic characteristics of each profile allows for the adaptation of the design of the MOOC to match the patterns of use by students, with the aim of increasing the completion rates of the courses. The user profiles that have been identified to date are based on the level of engagement of MOOC participants and their preference for one type of activities. The objective of this study is to define a series of MOOC participant profiles based on the temporary use of the course, considering the number of sessions and the scheduling performed by the participants, with the objective of contrasting the features of each type of student. With this objective, the information contained in the log files generated during the development of the third edition of the MOOC "Inglés Profesional / Professional English" has been used. Based on the results obtained, it can be concluded that the inclusion into any of the different profiles which successfully complete the MOOC does not imply a better performance in the course or a higher grade.
\end{abstract}

Keywords: MOOC; LMOOC; language MOOC; second language instruction; online courses; learner engagement.

En la sociedad actual, con una competencia en el mercado laboral mayor que en las anteriores décadas y en la que la necesidad de formación a lo largo de la vida es un imperativo, los MOOC parecen constituir la herramienta adecuada para la adquisición de nuevos conocimientos y habilidades, especialmente por parte de un creciente número de individuos cuyos objetivos incluyen la ampliación de la formación académica y el avance de la carrera profesional.

Los MOOC son cursos en línea de entre 4 y 10 semanas, sin restricción de acceso y gratuitos, lo cual permite que puedan ser seguidos por miles de estudiantes al mismo tiempo (Haggard, 2013). De hecho, es este gran número de estudiantes (potencialmente ilimitado) lo que diferencia los MOOC de otros medios de enseñanza en línea (Castrillo, 2014).

El primer MOOC estuvo basado en los principios del conectivismo, cuyo objetivo es mejorar la calidad de la educación a través del fortalecimiento de los vínculos horizontales y la estimulación de la cooperación conjunta en grupos de estudiantes (Seidametova, 2018). Los MOOC conectivistas, o cMOOC, se basan por lo tanto en la apertura y la enseñanza participativa (Jacoby, 2014), mientras que los xMOOC están basados en la absorción de contenidos y cuentan con un modelo de evaluación muy parecido a las clases tradicionales (Vázquez, Méndez, Román y López-Meneses, 2013). Algunos autores incluyen un tercer tipo, el tMOOC o MOOC basado en tareas, un híbrido de los dos anteriores. El tMOOC está compuesto por un cierto número de tareas que es obligatorio completar para poder seguir avanzando en el curso (Martí, 2012). Entre las numerosas categorías que se han acuñado 
recientemente se encuentran los MOOC para la enseñanza de idiomas o Language MOOC (LMOOC), que como se define en Wikipedia (s.f.), son cursos que permiten desarrollar de manera autónoma las habilidades en un idioma extranjero ("MOOC para la enseñanza de idiomas”). Según Martín-Monje y Bárcena (2014), los LMOOC son cursos dedicados en línea para segundos idiomas, con acceso sin restricciones y una participación potencialmente ilimitada, que pueden ser diseñados de manera efectiva para facilitar el desarrollo de las competencias lingüísticas comunicativas en grupos potencialmente masivos y altamente heterogéneos, cuyo principal interés compartido es aprender una lengua extranjera. Los LMOOC constituyen una combinación de prácticas y herramientas que permiten fomentar el uso del idioma por parte de los estudiantes de manera auténtica y significativa (Sokolik, 2015).

Los LMOOC se presentan como un campo de estudio incipiente, con escasa bibliografía escrita hasta la fecha. Dos de los trabajos más importantes son los monográficos editados por Martín-Monje y Bárcena (2014) y por Dixon y Thomas (2015), los cuales se han convertido en obras de referencia en el estudio de los LMOOC. Uno de los referentes clave en el diseño de LMOOC es el realizado por GimenoSanz, Navarro-Laboulais y Despujol-Zabala (2017) en su curso 'Learn Spanish: Basic Spanish for English Speakers', con 150.000 participantes en su primera edición.

Las particularidades de los LMOOC presentan una serie de dificultades. Un análisis de 77 LMOOC realizado por Heinsch y Rodríguez Pérez (2015, p. 8)

pone de manifiesto un modelo de enseñanza poco innovador, con numerosas similitudes con el modelo tradicional de enseñanza de idiomas, en el que se prioriza la adquisición de contenidos gramaticales sobre el desarrollo de estrategias para fomentar la interacción entre los participantes y de competencias comunicativas.

Sin embargo, incluso en aquellos LMOOC que fomentan la interacción entre estudiantes mediante tareas de evaluación por pares y aunque la actitud de los estudiantes es inicialmente positiva, existe una clara tendencia a sentirse intimidados ante ejercicios orales, como enviar grabaciones de sus producciones orales (Bárkányi, 2018). A estos obstáculos se une uno de los principales inconvenientes de los MOOC: la elevada tasa de abandono, la cual oscila entre el 75 y el 95\% (Poy y GonzalesAguilar, 2014) a pesar de que la calidad pedagógica de los MOOC se sitúa por encima de la media (Duart, Roig-Vila, Mengual-Andrés y Maseda Durán, 2017).

Una de las claves en la reducción de los problemas particulares de los LMOOC y de las tasas de abandono en estos cursos es el avance en la investigación sobre los hábitos de utilización e interacción con los MOOC por parte de los usuarios. Según Maseleno et al. (2018), Learning Analytics (LA) es la aplicación de técnicas analíticas en el análisis de datos educativos, tales como las actividades del alumno y la identificación de patrones de comportamiento con el objetivo de mejorar el aprendizaje. De hecho, según León Urrutia, Vázquez Cano y López-Meneses (2017), LA es una de las tendencias de futuro en educación más citadas en los informes internacionales de los últimos años. Las técnicas de LA posibilitan la identificación de puntos de intervención en los que los estudiantes dejan de participar y corren 
el riesgo de abandonar el curso (Anderson y Gavan, 2016). Un conocimiento más profundo y exacto de las características de la interacción de los participantes de los cursos con los distintos tipos de contenidos de los mismos permite la implementación y el diseño de modelos de MOOC que se adapten a las preferencias individuales de los estudiantes y acrecienten el grado de compromiso y vinculación de los participantes con el curso. Por este motivo, en los últimos años se ha incrementado el número de estudios que utilizan diversas técnicas de LA para analizar los datos generados por los MOOC. Uno de los estudios más citados de los últimos años, según el análisis de publicaciones llevado a cabo por Khalid y Ebner (2016), es el realizado por Kizilcec, Piech, y Schneider (2013) y mediante el cual identificaron cuatro perfiles de usuarios de MOOC, que se detallarán más adelante. Dentro del campo de los LMOOC, cabe destacar el estudio pionero llevado a cabo por Martín-Monje, Castrillo y MañanaRodríguez (2017), basado en el análisis estadístico inferencial de la interacción en línea de los estudiantes de un LMOOC y de los perfiles y los tipos de objetos de aprendizaje más fuertemente relacionados con la finalización con éxito del curso. Lee, Kadel, Madden y Gazi (2018) también emplearon LA en el análisis de un LMOOC en Coursera para demostrar la importancia del visionado de videos, la realización de tests y la evaluación entre pares en los resultados de los LMOOC.

La presente investigación analiza los datos obtenidos de la tercera edición del LMOOC "Inglés Profesional / Professional English" ofertado en la plataforma UNED Abierta, en el que se inscribieron un total de 8326 participantes. El objetivo de este trabajo es la identificación de perfiles de los alumnos trazando patrones temporales de acceso a los contenidos del MOOC. Para ello se analizarán, en primer lugar, los perfiles de usuarios identificados hasta la fecha en la literatura científica. En la siguiente sección se procederá a una descripción de la estructura del LMOOC "Inglés Profesional / Professional English" para pasar en el siguiente apartado a explicar el método utilizado para la extracción de la información necesaria para el trazado de los diferentes perfiles temporales. Posteriormente se procederá a la identificación de los perfiles de usuarios y el estudio comparativo de los mismos, basado en métodos cuantitativos. El último apartado recoge las conclusiones y las posibles ampliaciones del trabajo en el futuro.

\section{PERFILES DE PARTICIPANTES DE LOS MOOC}

En la literatura científica existen varias clasificaciones de los perfiles de los usuarios de los MOOC en función del nivel de interacción con los contenidos y el grado de compleción de las tareas y los objetivos del curso. Para el caso de los cMOOC, Milligan, Littlejohn y Margaryan (2013) establecen tres perfiles principales de participantes: (1) activos, aquellos que siguieron el curso y lo complementaron con blogs y cuentas en Twitter; (2) merodeadores, usuarios que siguieron el curso, pero no interaccionaron; y (3) pasivos, los que se sintieron insatisfechos con el MOOC pero que continuaron hasta el final del mismo. 
Hill (2013) identifica cinco tipos de usuarios de los MOOC: (1) ausentes son aquellos que se registran, pero no llegan a acceder al curso; (2) los observadores acceden al contenido, pero no realizan las actividades de evaluación; (3) drop-ins son quienes realizan algún tipo de actividad; (4) los pasivos completan el contenido pero no las actividades; (5) y los activos son aquellos que se involucran de lleno con el MOOC. Alario-Hoyos, Pérez-Sanagustín, Delgado-Kloos, Parada y MuñozOrganero (2014) condensan el tipo de participantes en tres grupos: merodeadores, aquellos alumnos que se registran pero que a lo sumo consultan un número reducido de recursos; estudiantes que no completan el curso, y participantes que completan el curso. A partir de estas categorías generales, estos autores identifican siete patrones, algunos de los cuales coinciden con las categorías de Hill: (1) ausentes; (2) observadores; (3) drop-ins; (4) latecomers, que, al igual que los drop-ins, no completan el curso, pero con el condicionante de haberse registrado una vez iniciado el MOOC; (5) drop-in latecomers son quienes se unen al curso una vez iniciado y lo abandonan antes de que termine, sin haberlo completado; (6) participantes no comprometidos, aquellos que completan el curso sin realizar actividades que requieran una carga de trabajo importante; y (7) comprometidos, que completan tanto el curso como las actividades.

De manera similar, Hill, Grünewald et al. (2013) identifican cinco perfiles de usuarios en función a su nivel de interacción en los foros del MOOC: (1) inactivos, aquellos que no visitan nunca el foro; (2) pasivos, usuarios que solo consumen información; (3) reacting, que complementan aspectos de cuestiones ya planteadas; (4) acting, quienes plantean cuestiones y lideran las discusiones; y (5) supervisores/ sustentadores, que además de liderar las discusiones, resumen el conocimiento adquirido. Anderson, Huttenlocher, Kleinberg y Leskovec (2014) también identifican cinco formas de compromiso con los MOOC por parte de los estudiantes: (1) espectadores, que visualizan las clases, pero apenas entregan alguna tarea; (2) resolventes, quienes entregan tareas pero apenas visualizan las clases; (3) versátiles, que mantienen un equilibrio entre el visualizado de las clases y las tareas; (4) coleccionistas, que descargan los vídeos de las clases pero apenas entregan tareas; y (5) bystanders, quienes apenas realizan actividad en el curso.

Por último, Kizilcec, Piech, y Schneider (2013) identifican cuatro perfiles en función del nivel de compromiso con el MOOC y el grado de participación: (1) acabadores, estudiantes que al menos intentaron realizar todas las tareas; (2) asistentes, estudiantes que no realizaron apenas tareas pero que visionaron los vídeos; (3) desconectados, estudiantes que realizaron las tareas al comienzo del curso, pero que en algún momento se desvincularon del MOOC; y 4) sampling, estudiantes que ven vídeos, normalmente solo uno, durante uno o dos periodos de evaluación.

Si bien las distintas clasificaciones de perfiles nos aportan información valiosa en cuanto al grado de vinculación y compromiso de los participantes de los MOOC y del tipo de contenido preferido por los mismos, no se ha encontrado ninguna 
clasificación relativa a patrones temporales de rutinas de utilización y acceso a los cursos. De este modo, un usuario que complete el curso en una sola sesión, que realice todas las tareas y que participe en los foros sería considerado como un usuario activo por todas las clasificaciones anteriores, al igual que un usuario que programe la realización del curso dividiéndolo en sesiones más cortas extendidas en el tiempo, aunque claramente se trate de perfiles de estudiantes con características y preferencias de aprendizaje distintas.

\section{DESCRIPCIÓN DEL MOOC}

La tercera edición del MOOC "Inglés Profesional / Professional English" tuvo una duración de tres semanas, del 18 de abril al 9 de mayo de 2017. Sin embargo, el acceso a los foros quedó habilitado hasta el 22 de mayo, pero sin apoyo por parte del equipo docente ni posibilidad de realizar nuevas inscripciones en el curso, aunque los usuarios ya registrados podían acceder al foro y completar actividades. El número total de inscritos en el MOOC fue de 8326.

El curso estuvo dividido en diferentes secciones a las que se accedía a través de cinco pestañas localizadas en la barra superior de la página. La primera pestaña, 'Inicio', contenía una página que ofrecía información básica sobre el MOOC, como la identificación del equipo docente, los objetivos del curso o los requisitos previos. La segunda pestaña, 'Curso', proporcionaba acceso al contenido fundamental del MOOC y se presentaba en forma de página web navegable con botones de avance y retroceso, así como una barra de menú lateral que permitía el acceso a todos los módulos del curso. El primer bloque del menú lateral estaba formado por una guía inicial dividida en tres apartados con información práctica sobre el uso de los foros, las actividades de autoevaluación y el visionado efectivo de los vídeos. El segundo bloque del menú lateral dirigía a un test de conocimientos previos compuesto por 20 preguntas, cuyo objetivo era garantizar que el nivel del alumno se correspondía con el nivel B1 del Marco Común Europeo para las Lenguas. El tercer bloque del menú lateral estaba denominado ‘Bloque o’ o bloque de Introducción. En el primer apartado del bloque se incluía un vídeo de 5 minutos y medio de duración con información relativa a la organización y desarrollo del curso. El segundo apartado dirigía a la sección del foro correspondiente a información general. El tercer apartado introducía una actividad especial voluntaria que no se tomaba en cuenta para el cálculo de la calificación final del curso. Dicha actividad se basaba en la descarga y utilización de una aplicación de podcasts ${ }^{1}$. Los siguientes tres bloques del menú lateral incluían el material fundamental del curso, dividido en tres módulos con una estructura paralela. Cada bloque estaba formado por una página en la que se enunciaban los objetivos de aprendizaje del módulo, seguida de cuatro vídeos distintos con una casilla de confirmación que el estudiante debía marcar tras el completo visionado de los mismos. Posteriormente se ofrecía un test de evaluación compuesto por 20 preguntas, un test de comprensión oral compuesto de un archivo 
de audio y 10 preguntas relativas al mismo, además de un último apartado que dirigía a la sección del foro correspondiente al módulo en cuestión. La duración total de los cuatro vídeos de cada uno de los módulos era de 7 minutos y 22 segundos para el primero, 11 minutos y 16 segundos para el segundo y 6 minutos y 31 segundos para el tercero. La duración de los archivos de audio para el test de comprensión oral de cada módulo era de 2 minutos y 39 segundos para los dos primeros módulos y 2 minutos y 50 segundos para el tercero. La última sección del menú lateral se correspondía con un test final de evaluación compuesto por 20 preguntas. La siguiente tabla muestra un resumen del conjunto de actividades incluidas en el curso.

Tabla 1. Resumen de las actividades del curso

\begin{tabular}{|l|l|l|l|l|l|}
\hline Módulo 1 & Objetivos & 4 vídeos (7' 22”) & Test 20 preguntas & $\begin{array}{l}\text { Test oral (2’39”) 10 } \\
\text { preguntas }\end{array}$ & $\begin{array}{l}\text { Foro } \\
\text { módulo 1 }\end{array}$ \\
\hline Módulo 2 & Objetivos & 4 vídeos (11' 16”) & Test 20 preguntas & $\begin{array}{l}\text { Test oral (2' 39”) 10 } \\
\text { preguntas }\end{array}$ & $\begin{array}{l}\text { Foro } \\
\text { módulo 2 }\end{array}$ \\
\hline Módulo 3 & Objetivos & 4 vídeos (6' 31”) & Test 20 preguntas & $\begin{array}{l}\text { Test oral (2’ 50”) 10 } \\
\text { preguntas }\end{array}$ & $\begin{array}{l}\text { Foro } \\
\text { módulo 3 }\end{array}$ \\
\hline
\end{tabular}

Test final (20 preguntas)

La tercera pestaña, 'Programa', consistía en una sola página que resumía el contenido de los tres módulos principales del curso y los métodos de evaluación. La cuarta pestaña, 'Foro', proporcionaba acceso a una serie de foros moderados por los miembros del equipo docente, divididos en cuatro secciones: una general para cuestiones relativas al curso y tres secciones más dedicadas a los contenidos de cada uno de los módulos principales. Por último, la pestaña 'Progreso' mostraba el avance individual del estudiante en el MOOC dividiendo la información de dos modos distintos. Por un lado, un gráfico de barras ofrecía información estadística de compleción de cada una de las actividades del curso. Por otro lado, los distintos módulos y submódulos eran presentados en forma de lista con hipervínculos que mostraban la puntuación asignada a cada una de las tareas de evaluación de los correspondientes submódulos. En la parte superior de esta pestaña se mostraba un botón que dirigía a una página que permitía generar una acreditación de compleción del curso si se cumplían los requisitos de evaluación determinados.

\section{METODOLOGÍA}

El análisis de los datos de interacción de los usuarios con el MOOC se ha realizado mediante un programa ad hoc diseñado para realizar una doble función. Por un lado, el programa extrae la información relevante del registro de logs de los usuarios de la plataforma. Por otro lado, a partir de un fichero csv (comma separated values) generado con la información almacenada en la base de datos, el programa obtiene 
la información relativa a la puntuación obtenida por los usuarios en cada una de las pruebas de evaluación del curso y a su elegibilidad para la solicitud de la acreditación de compleción del MOOC.

La actividad de los usuarios del curso se registró en el servidor de la plataforma mediante una serie de ficheros de $\log$ que contienen en cada entrada un objeto JSON (JavaScript Object Notation), el cual es un formato de texto ligero para el intercambio de datos. El objeto JSON, por su parte, contiene una serie de campos relativos al acceso por parte del usuario a los contenidos del curso. La información que se ha extraído de dichos objetos para el presente análisis ha sido el nombre de usuario, la URL del recurso y la fecha y hora de acceso.

El archivo csv contiene una tabla, obtenida a partir de la información almacenada en la base de datos de la plataforma del curso, que relaciona el nombre del usuario con la puntuación obtenida en cada una de las pruebas de evaluación del curso. De manera adicional, la tabla contiene una columna que indica si el usuario es elegible para la generación y envío de una acreditación de compleción del curso.

Mediante la creación de un programa en el lenguaje de programación Java, se ha procedido a extraer la información pertinente tanto de los ficheros de log como del archivo csv y se ha generado una lista de objetos que almacenan la información relativa a cada uno de los usuarios. Dichos objetos contienen una lista de sesiones que se corresponden a las sesiones individuales de acceso a la plataforma del MOOC por parte de cada uno de los usuarios. Para cada una de las sesiones se almacena el tipo de recursos al que el usuario ha accedido y el tiempo de inicio y finalización de cada sesión. De manera adicional, se almacena para cada uno de los usuarios su puntuación en cada una de las pruebas de evaluación y si el usuario es elegible para la acreditación y, por lo tanto, ha completado el curso.

El programa informático diseñado, después de procesar tanto los ficheros de texto que contienen los logs, como el archivo csv, genera una serie de estadísticas que, como se analizará en la siguiente sección, permite la identificación de una serie de perfiles de usuario en función de patrones temporales de acceso al curso.

\section{ANÁLISIS DE LOS DATOS}

Tras el procesamiento de los datos de acceso de los usuarios de la plataforma se han identificado cinco perfiles de usuario:

1. Pre-consumidores. Usuarios que nunca acceden a los contenidos principales del MOOC, esto es, a ninguno de los tres módulos en que está dividido el curso. Este grupo se correspondería con los ausentes de Hill. La interacción que estos usuarios realizan con el MOOC es mínima, por lo que no aportan información sustancial para el trazado de perfiles temporales de uso del MOOC.

2. Usuarios intensivos. Son usuarios que acceden a los contenidos principales del curso en el transcurso de una o dos sesiones. 
3. Usuarios rutinarios. Usuarios que acceden a los contenidos principales del curso en el transcurso de más de dos sesiones comprendidas dentro de la misma franja horaria ${ }^{2}$.

4. Usuarios flexibles. Usuarios que acceden a los contenidos principales del curso en el transcurso de más de dos sesiones que no pertenecen a la misma franja horaria.

5. Usuarios exhaustivos. Usuarios que acceden al curso mediante más de quince sesiones.

Tabla 2. Perfiles de usuario

\begin{tabular}{|c|l|}
\hline Pre-consumidores & • Sin acceso a contenidos principales \\
\hline Intensivos & • Una o dos sesiones \\
\hline Rutinarios & $\bullet$ Más de 2 sesiones en la misma franja \\
\hline Flexibles & $\bullet$ Más de 2 sesiones en distinta franja \\
\hline Exhaustivos & $\bullet$ Más de 15 sesiones \\
\hline
\end{tabular}

Figura 1. Distribución de Perfiles de Usuario

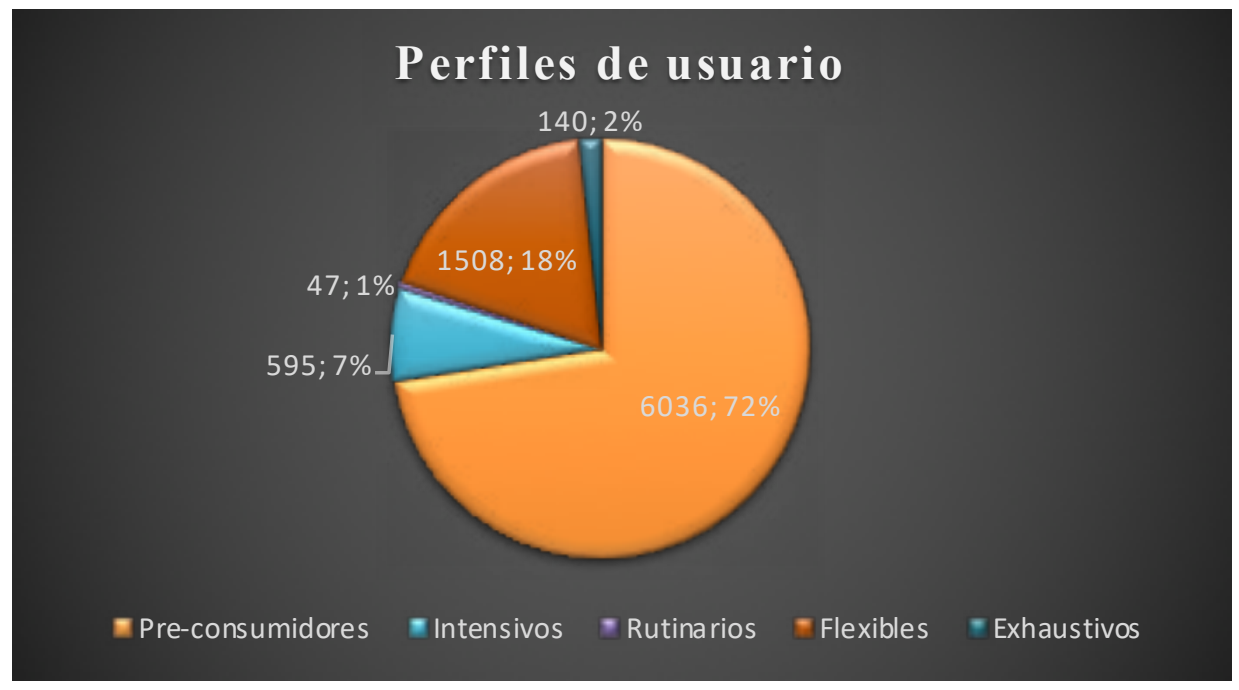


De entre el primer tipo de usuarios, los que no llegan a acceder al contenido principal del curso o pre-consumidores, podemos distinguir varios grupos, como puede observarse en la figura 2:

1. Un primer grupo está formado por los alumnos que se inscriben pero que no llegan a acceder al curso en ningún momento. Este grupo está identificado como "solo inscritos" en la figura 2 y está formado por un total de 4275 alumnos de los 8326 inscritos en el curso, lo que supone un 51,34\% del total.

2. Un segundo grupo está formado por alumnos que no acceden a ninguno de los módulos del curso (ni a los módulos iniciales, ni a los módulos principales, ni a los test de evaluación), pero sí a los foros. Son un total de 43 alumnos, identificados como "solo a foros" en la figura 2, que representan el o,52\% del total de 8326 inscritos.

3. Un tercer grupo está integrado por dos subgrupos de alumnos: por un lado, los 1124 alumnos que acceden a alguna de las secciones explicativas o "módulos iniciales" del MOOC, y por otro, los 594 alumnos que realizan el "test previo", como puede observarse en la figura 2. Estos 1718 individuos, que representan un $20,63 \%$ del total de 8326 inscritos, son por lo tanto alumnos que interaccionan con el curso, pero que no llegan a acceder a ninguno de los tres bloques de contenido principal del MOOC.

Figura 2. Acceso a recursos de Pre-consumidores

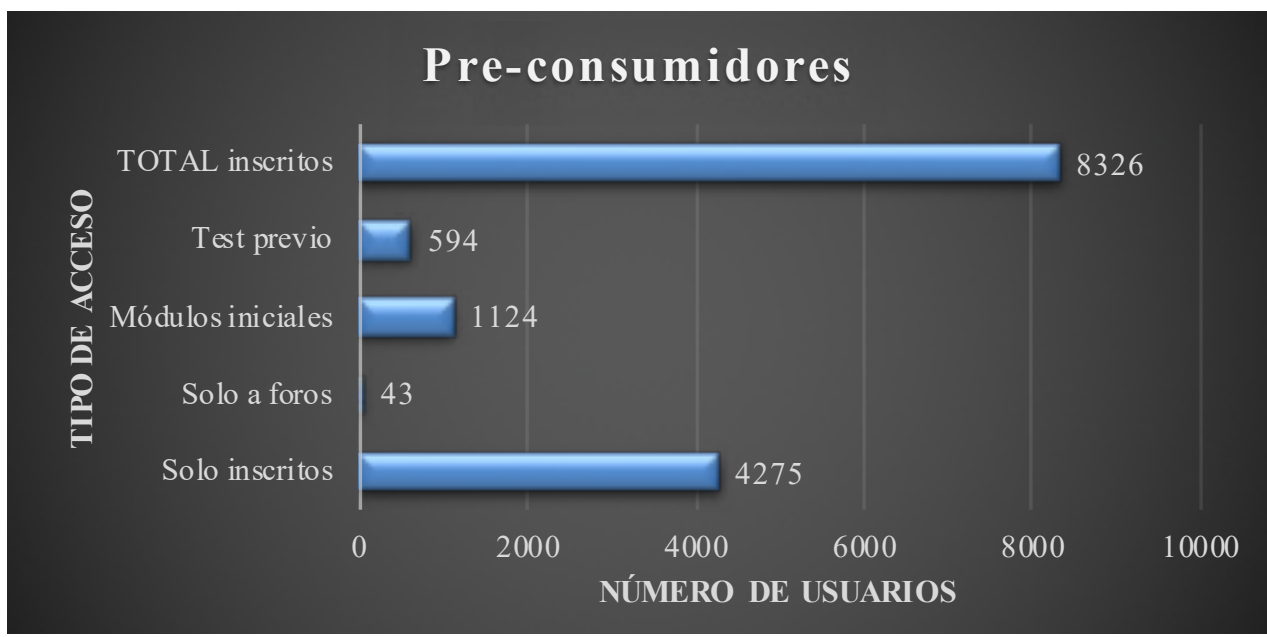


Entre los grupos de usuarios intensivos, rutinarios, flexibles y exhaustivos se identifican una serie de rasgos característicos en función del tiempo invertido en el curso, el número total de sesiones y el número de interacciones en los foros.

Como puede observarse en la tabla 3 , los usuarios intensivos son los que dedican una menor cantidad de tiempo en el curso, una media de 34,14 minutos, mientras que los exhaustivos dedican una media de 369,75. Los usuarios flexibles dedican más tiempo de media al MOOC que los usuarios rutinarios, 100,51 minutos frente a 137,13 .

Tabla 3. Estadísticas descriptivas de los minutos totales invertidos en el MOOC

\begin{tabular}{|l|c|c|c|c|}
\hline & Intensivos & Rutinarios & Flexibles & Exhaustivos \\
\hline Media & 34,14 & 100,51 & 137,13 & 369,75 \\
\hline Desviación típica & 36,65 & 76,11 & 108,73 & 223,55 \\
\hline
\end{tabular}

Según puede observarse, la desviación típica es alta, especialmente en el caso de los usuarios intensivos. Esto se debe al efecto de los usuarios que no completan el curso y no son elegibles para la obtención del certificado, entre los que encontramos una dispersión extrema de los datos, como puede observarse en la tabla 4, que muestra el rango de los datos para dichos usuarios. Los valores bajos en los minutos totales invertidos en el curso son esperables, ya que el tiempo dedicado por los estudiantes que no completan el curso es lógicamente menor al de los que sí lo completan. Sin embargo, una explicación para los valores elevados de la duración de las sesiones puede ser el hecho de que los estudiantes que no completan el curso no estén focalizados plenamente en el curso, sino que estén realizando varias tareas en paralelo con el dispositivo electrónico, por ejemplo, consultando otras páginas web, lo cual produciría el efecto de alargar artificialmente las sesiones.

Tabla 4. Rango de los minutos totales invertidos en el MOOC por estudiantes que no completan el curso

\begin{tabular}{|l|c|c|c|c|}
\hline & Intensivos & Rutinarios & Flexibles & Exhaustivos \\
\hline Rango & 173 & 253 & 488 & 946 \\
\hline
\end{tabular}

Si consideramos solo los usuarios elegibles para la obtención del certificado, encontramos una menor dispersión de los datos, como se muestra en la tabla 5. 
Tabla 5. Estadísticas descriptivas de los minutos totales invertidos en el MOOC por estudiantes elegibles para el certificado

\begin{tabular}{|l|c|c|c|c|}
\hline & Intensivos & Rutinarios & Flexibles & Exhaustivos \\
\hline Media & 141 & 150,93 & 238,78 & 458,24 \\
\hline Desviación típica & 72,13 & 65,93 & 101,97 & 184,14 \\
\hline
\end{tabular}

Respecto al número de sesiones, los usuarios flexibles dedican una media de 6,38 sesiones al curso, mientras que los usuarios rutinarios dedican una media de 3,79 , como se muestra en la tabla 6. Los usuarios intensivos son los usuarios que dedican una o dos sesiones al MOOC. De hecho, el 44\% le dedica solo una sesión y el $56 \%$ restante le dedica dos sesiones. La media de los usuarios exhaustivos, aquellos que dedican más de 15 sesiones, es de 26,3 sesiones.

Tabla 6. Estadísticas descriptivas de las sesiones totales invertidas en el MOOC

\begin{tabular}{|l|c|c|c|c|}
\hline & Intensivos & Rutinarios & Flexibles & Exhaustivos \\
\hline Media & 1,56 & 3,79 & 6,38 & 26,3 \\
\hline Desviación típica & 0,5 & 1,04 & 3,2 & 15,0 \\
\hline
\end{tabular}

De nuevo, si eliminamos los usuarios que no completan el curso encontramos una menor dispersión de datos y, en todos los casos excepto en el de los usuarios exhaustivos, encontramos un incremento en la media de sesiones, como se muestra en la tabla 7. De hecho, en el caso de los alumnos intensivos que completan el curso, el $85 \%$ lo hace en 2 sesiones, mientras que solo el 15\% realiza todo el curso en una única sesión. El que la media del número de sesiones de los usuarios exhaustivos sea mayor cuando se incluye a aquellos que no han completado el curso puede deberse a alumnos que siguen consultando el MOOC una vez finalizado y que contemplan el curso como material de consulta, sin intención de realizar las tareas de evaluación del mismo.

Tabla 7. Estadísticas descriptivas de las sesiones totales invertidos en el MOOC por estudiantes elegibles para el certificado

\begin{tabular}{|l|l|l|l|l|}
\hline & \multicolumn{1}{|c|}{ Intensivos } & \multicolumn{1}{c|}{ Rutinarios } & \multicolumn{1}{c|}{ Flexibles } & \multicolumn{1}{c|}{ Exhaustivos } \\
\hline Media & 1,85 & 4,14 & 8,13 & 23,37 \\
\hline Desviación típica & 0,37 & 1,29 & 3,38 & 8,31 \\
\hline
\end{tabular}

En consecuencia, y como podemos observar en la tabla 8, que muestra la duración media de las sesiones entre los usuarios que completan el MOOC, los usuarios 
intensivos tienden a realizar sesiones de estudio más largas, algo que es previsible, mientras que los usuarios con sesiones más cortas son los usuarios exhaustivos.

Tabla 8. Estadísticas descriptivas del número de minutos por sesión por los usuarios que completan el MOOC

\begin{tabular}{|l|c|c|c|c|}
\hline & Intensivos & Rutinarios & Flexibles & Exhaustivos \\
\hline Media & 76,27 & 37,33 & 32,3 & $\mathbf{2 0 , 5 2}$ \\
\hline Desviación típica & 34,99 & 15,03 & 14,53 & 8,21 \\
\hline
\end{tabular}

En cuanto al acceso al foro, podemos observar una mayor actividad entre los usuarios exhaustivos, seguido por los usuarios flexibles, como se observa en la tabla 9, lo cual es lógico, ya que son los perfiles que presentan una mayor duración total de la interacción con el curso.

Tabla 9. Estadísticas descriptivas del acceso a los foros por los usuarios del MOOC

\begin{tabular}{|l|c|c|c|c|}
\hline & Intensivos & Rutinarios & Flexibles & Exhaustivos \\
\hline Media & $\mathbf{1 , 8 6}$ & 7,81 & 9,81 & $\mathbf{2 8 , 6 6}$ \\
\hline Desviación típica & 4,03 & 9,91 & 15,74 & 47,1 \\
\hline
\end{tabular}

Sin embargo, como podemos observar, para todos los perfiles existe una enorme dispersión de los datos de interacción con los foros, que continúa en valores elevados al eliminar a los usuarios que no completan el curso, como se muestra en la tabla 10. La alta dispersión indica la presencia de un cierto número de usuarios que hace un uso extensivo del foro. El que la desviación típica sea alta, cercana a la media en todos los perfiles excepto en el exhaustivo, donde el valor es incluso superior, indica que la presencia de participantes con altos niveles de intervención en los foros no es un rasgo definitorio del perfil, sino que encontramos usuarios especialmente activos en los foros dentro de todos los perfiles.

Tabla 10. Estadísticas descriptivas de las sesiones totales invertidos en el MOOC por estudiantes elegibles para el certificado

\begin{tabular}{|l|c|c|c|c|}
\hline & Intensivos & Rutinarios & Flexibles & Exhaustivos \\
\hline Media & 9,6 & 15,64 & 18,94 & 39,71 \\
\hline Desviación típica & 8,48 & 12,49 & 21,92 & 54,48 \\
\hline
\end{tabular}

Por último, en la tabla 11 observamos como la nota final (promediada sobre 10) no depende del perfil, ya que encontramos valores uniformes para los cuatro perfiles 
y valores de dispersión mínimos. Este hecho es interesante por no predecible, ya que una mayor implicación temporal en el curso debería llevar aparejada una calificación mayor en la nota global.

Tabla 11. Estadísticas descriptivas de las sesiones totales invertidos en el MOOC por estudiantes elegibles para el certificado

\begin{tabular}{|l|c|c|c|c|}
\hline & Intensivos & Rutinarios & Flexibles & Exhaustivos \\
\hline Media & 9,02 & 9,16 & 9,05 & 9,08 \\
\hline Desviación típica & 0,08 & 0,07 & 0,07 & 0,07 \\
\hline
\end{tabular}

\section{CONCLUSIONES}

El presente estudio supone una primera aproximación al perfilado de usuarios en función a patrones temporales de interacción con los MOOC mediante los datos recopilados del LMOOC Inglés Profesional / Professional English”. Se han comparado los rasgos de los cuatro perfiles con actividad en el curso y se han identificado diferencias en cuanto al número de sesiones y minutos totales dedicados y en cuanto al uso de los foros. Sin embargo, se ha comprobado que la pertenencia a uno u otro perfil no implica un mayor desempeño en el curso y una nota total mayor.

Una de las posibles cuestiones de estudio que se plantean a partir de los resultados es por qué los perfiles que dedican más tiempo y más sesiones al estudio del MOOC e interaccionan más en los foros no obtienen una calificación mayor que perfiles como el intensivo, con una implicación temporal mínima. Uno de los posibles objetivos a la hora de diseñar un MOOC podría ser la inclusión de actividades que supongan una profundización en los contenidos y una mayor maestría por parte de los perfiles que dedican más tiempo a la realización de los MOOC, la cual debería verse reflejada en la nota final.

Por otro lado, el LMOOC "Inglés Profesional / Professional English" tuvo una duración de tres semanas, lo cual lo convierte en un MOOC de corta duración ${ }^{3}$. Una de las posibilidades de ampliación del estudio es analizar el desempeño para cada uno de los perfiles identificados en un MOOC de mayor duración. Este análisis sería de especial interés en el caso de los usuarios intensivos, que a priori son los que deberían verse más afectados por una duración mayor de los cursos. De manera adicional, sería relevante analizar los patrones temporales de uso en función del momento de inicio de la actividad en el MOOC, de tal forma que pudiera determinarse si un inicio tardío del curso favorece la adopción de perfiles como el intensivo y el flexible en detrimento del perfil rutinario.

Por último, es necesario tener en cuenta las limitaciones de la investigación debidas a las particularidades del curso del que se han obtenido los datos: un LMOOC de 3 semanas de duración orientado principalmente a hispanohablantes. Para que los resultados del presente estudio puedan extrapolarse a otros tipos de 
MOOC sería necesario realizar trabajos más exhaustivos que lograran identificar la misma tipología de perfiles de participantes en MOOC de distintas temáticas y características.

\section{NOTAS}

1. Debido a que dicha aplicación es un módulo ajeno al MOOCy, por motivo de limitaciones técnicas, no ha sido posible el acceso a los logs de la aplicación, la interacción con dicha aplicación no se ha tenido en cuenta para el análisis de los datos de uso de los usuarios y el consiguiente trazado de los perfiles.

2. Se ha considerado que dos sesiones pertenecen a la misma franja horaria cuando, bien entre el inicio de ambas o bien entre la conclusión de ambas, no se supera el intervalo temporal de 90 minutos.

3. Se ha demostrado que la duración de los primeros MOOC (entre 6 y 8 semanas) era demasiado larga, por lo que se aconseja una duración de 3 a 4 semanas y el desarrollo de un módulo temático por semana (Castrillo, Martín-Monje y Vázquez-Cano, 2018).

\section{REFERENCIAS}

Alario-Hoyos, C., Pérez-Sanagustín, M., Delgado-Kloos, C., Parada, H. A., y Muñoz-Organero, M. (2014). Delving into Participants' Profiles and Use of Social Tools in MOOC. IEEE Transactions on Learning Technologies 7(3), 260-266. doi: 10.1109/TLT.2014.2311807.

Anderson, M., y Gavan, C. (2016). Developing Effective Educational Experiences through Learning Analytics. Hershey, PA: IGI Global.

Anderson, A., Huttenlocher, D., Kleinberg, J., y Leskovec, J. (2014). Engaging with massive online courses. Proceedings of the 23rd International Conference on World wide web (pp. 687-698). New York: ACM. doi: 10.1145/2566486.2568042.

Bárkányi, Z. (2018). Can you teach me to speak? Oral practice and anxiety in a language MOOC. En F. Rosell-Aguilar, T. Beaven y M. F. Gutiérrez (Eds.), Innovative language teaching and learning at university: integrating informal learning into formal language education (9-16). Research-publishing. net. doi: 10.14705/rpnet.2018.22.771.
Castrillo, M. D. (2014). Language Teaching in MOOC: the Integral Role of the Instructor. En E. Martín-Monje y E. Bárcena (Eds.), Language MOOC. Providing Learning, Transcending Boundaries (67-90). Berlin: De Gruyter Open.

Castrillo M. D., Martín-Monje, E., y Vázquez-Cano, E. (2018). Guía práctica para el diseño y tutorización de MOOC. MiríadaX. Recuperado de https://bit. ly/2BXpSpW

Dixon, E., y Thomas, M. (Eds.). (2015). Researching language learner interactions online: From social media to MOOCs. San Marcos, Texas: Computer Assisted Language Instruction Consortium.

Duart, J. M., Roig-Vila, R., Mengual-Andrés, S., y Maseda Durán, M.-A. (2017). La calidad pedagógica delos MOOCa partir de la revisión sistemática de las publicaciones JCR | The pedagogical quality of MOOCs based on a systematic review of JCR and Scopus publications (2013-2015). Revista Española de Pedagogía, 75(266), 29-46. doi: doi.org/10.22550/REP75-1-2017-02. 
Gimeno-Sanz, A., Navarro-Laboulais, C., y Despujol-Zabala, I. (2017). Additional functionalities to convert an XMOOC into an xLMOOC. En C. Delgado Kloos, P. Jermann, M. Pérez-Sanagustín, D. Seaton y S. White (Eds.), Digital education: Out to the world and back to the campus. Proceedings of the European MOOC Stakeholders Summit 2017 (pp. 48-57). Cham, Swizterland: Springer.

Grünewald, F., Mazandarani, E., Meinel, C., Teusner, R., Totschnig, M., y Willems, C. (2013). OpenHPI - a Case-Study on the emergence of two learning communities. Proc. IEEE Global Eng. Edu. Conf. (pp. 13-15).

Haggard, S. (2013). The Maturing of the MOOC (Research No. 130). London: Department for Business Innovation \& Skills - UK Government. Recuperado de https://bit.ly/JVRD5c

Heinsch, B., y Rodríguez Pérez, M. N. (2015). MOOC: un nuevo escenario de enseñanzaaprendizaje de lenguas extranjeras. @tic. revista d'innovació educativa (14), 1-12. doi: 10.7203/attic.14.4315.

Hill, P. (2013, 10 de marzo). Emerging student patterns in MOOC: A (Revised) graphical view [Entrada de blog]. Recuperado de https://mfeldstein.com/ emerging-student-patterns-in-moocs-arevised-graphical-view/

Jacoby, J. (2014). The disruptive potential of the massive open online course: A literature review. Journal of Open, Flexible and Distance Learning, 18(1), $73-85$.

Khalil, M., y Ebner, M. (2016). What is learning analytics about? A survey of different methods used in 20132015. Proceedings of Smart Learning Conference, Dubai, UAE, March 7-9, 2016 (pp. 294-304). Dubai: HBMSU Publishing House.

Kizilcec, R. F., Piech, C., y Schneider, E. (2013). Deconstructing disengagement: Analyzing learner subpopulations in massive open online courses. 3rd International Conference on Learning Analytics and Knowledge (pp. 170-179). New York: ACM. doi: 10.1145/2460296.2460330.

Lee, S., Kadel, R. S., Madden, A., y Gazi, Y. (2018). How Can Learner Analytics Data Inform Language MOOC Design? Boğaziçi Üniversitesi Eğitim Dergisi, 35(1), 19-29. Recuperado de http://dergipark.gov.tr/ buje/issue/42479/513654

León Urrutia, M., Vázquez Cano, E., y LópezMeneses, E. (2017). MOOC learning analytics using real-time dynamic metrics. @tic. revista d'innovació educativa (18), 38-47. doi: 10.7203/attic.18.10022.

Martí, J. (2012, 24 de agosto). Tipos de MOOC [Entrada de blog]. Recuperado de http://www.xarxatic.com/tipos-deMOOC/

Martín-Monje, E., y Bárcena, E. (Eds.) (2014). Language MOOC. Providing Learning, Transcending Boundaries. Berlin: De Gruyter Open.

Martín-Monje, E., Castrillo, M. D., y MañanaRodríguez,J.(2017).Understandingonline interaction in language MOOCs through learning analytics. Computer Assisted Language Learning, 31(3), 251-272. doi: 10.1080/09588221.2017.1378237.

Maseleno, A., Sabani, N., Huda, M., Ahmad, R., Jasmi, K. A., y Basiron, B. (2018). Demystifying learning analytics in personalised learning. International Journal of Engineering \& Technology, $7(3), 1124-1129$.

Milligan, C., Littlejohn, A., y Margaryan, A. (2013). Patterns of engagement in connectivist MOOC. Journal of Online Learning \& Teaching 9(2), 149-159.

MOOC para la enseñanza de idiomas. (s.f.). En Wikipedia. Recuperado de https:// es.wikipedia.org/wiki/MOOC para la ense\%C3\%B1anza de idiomas

Poy, Y., y Gonzales-Aguilar, A. (2014). Factores de éxito de los MOOC: algunas consideraciones críticas. Revista 
Ibérica de Sistemas y Tecnologías de Información, SPE1 (2014), 105-118.

Seidametova, Z. (2018). Design and Development of MOOCs. En V. Ermolayev, M. C. Suárez-Figueroa, V. Yakovyna, V. Kharchenko, V. Kobets, H. Kravtsov, V. Peschanenko, Y. Prytula, M. Nikitchenko y A. Spivakovsky (Eds.), Proceedings of the 14th International Conference on ICT in Education, Research and Industrial Applications. Integration, Harmonization and Knowledge Transfer.
Volume II: Workshops (Vol. 2104, 462471). CEUR Workshop Proceedings.

Sokolik, M. (2014). What Constitutes an Effective Language MOOC? En E. Martín-Monje y E. Bárcena (Eds.), Language MOOC. Providing Learning, Transcending Boundaries (16-32). Berlin: De Gruyter Open.

Vázquez, E., Méndez, J. M., Román, P., y López-Meneses, E. (2013). Diseño y desarrollo del modelo pedagógico de la plataforma educativa Quantum University Project. Campus Virtuales, 2(1), 54-63.

\section{PERFIL ACADÉMICO Y PROFESIONAL DEL AUTOR}

Juan José del Peral Pérez. Graduado en Estudios Ingleses por la UNED e Ingeniero Técnico en Telecomunicaciones por la Universidad Carlos III de Madrid, Juan José del Peral es profesor de inglés de secundaria y realiza un Doctorado en Estudios Lingüísticos y Literarios: Teoría y Aplicaciones en la UNED. Su línea principal de investigación es el uso de las TIC en la enseñanza de idiomas.

E-mail: jdelperal2@alumno.uned.es

Dirección:

Dpto. Filologías Extranjeras y sus Lingüísticas

UNED

Paseo Senda del Rey, 7

28040 Madrid (España)

Fecha de recepción del artículo: 04/10/2018

Fecha de aceptación del artículo: 23/01/2019

Fecha de aprobación para maquetación: 24/02/2019 